\title{
Food allergy in infants assessed in two German birth cohorts 10 years after the EuroPrevall Study
}

\author{
Daniela V. Röhrl ${ }^{1}$ | Susanne Brandstetter ${ }^{2,3}$ | Linda P. Siziba ${ }^{4}$ | | \\ Dietrich Rothenbacher $^{5}$ | Vincent D. Gaertner ${ }^{1}$ | Susanne Harner ${ }^{1}\left(\right.$ I $_{\text {| }}$ \\ Angela Köninger $^{2,6}$ | Christian Apfelbacher ${ }^{2,7} \odot$ | Michael Melter ${ }^{2,3}$ | Jon Genuneit ${ }^{4,5}$ । \\ Michael Kabesch ${ }^{1,2}$ (1) | | the KUNO Kids Study Group
}

\footnotetext{
${ }^{1}$ Department of Pediatric Pneumology and Allergy, University Children's Hospital Regensburg (KUNO), of the University of Regensburg and the Order of St. John at the St. Hedwig Hospital, Regensburg, Germany

${ }^{2}$ Science and Innovation Campus Regensburg (WECARE) of the Order of St. John at the St. Hedwig Hospital, Regensburg, Germany

${ }^{3}$ University Children's Hospital Regensburg (KUNO) of the University of Regensburg and the Order of St. John at the St. Hedwig Hospital, Regensburg, Germany

${ }^{4}$ Pediatric Epidemiology, Department of Pediatrics, Medical Faculty, Leipzig University, Leipzig, Germany

${ }^{5}$ Institute of Epidemiology and Medical Biometry, Ulm University, Ulm, Germany

${ }^{6}$ Department of Obstetrics and Gynecology of the University of Regensburg and the Order of St. John at the St. Hedwig Hospital, Regensburg, Germany ${ }^{7}$ Institute of Social Medicine and Health Systems Research, Otto von Guericke University, Magdeburg, Germany
}

Correspondence

Michael Kabesch, Department of Pediatric

Pneumology and Allergy, University

Children's Hospital Regensburg (KUNO)

at the St. Hedwig Hospital of the Order

of St. John, Steinmetzstr. 1-3, Regensburg

D-93049, Germany.

Email: Michael.kabesch@barmherzige-

regensburg.de

\section{Funding information}

The KUNO Kids study is funded by research grants of the EU (HEALS:

603946) and the German Federal

Ministry for Education and Research

(SYSINFLAME: 01ZX1306E and

NAMIBIO: 01EA2108B). Further financial support was provided by the University Children's Hospital of the University of Regensburg and the Order of St. John. The funding bodies had neither influence on the design of the study, on the collection, analysis, and interpretation of data, nor in the writing of the manuscript. The UIm SPATZ Health Study was funded through an unrestricted grant by the Medical Faculty of UIm University and by the

\begin{abstract}
Background: The prevalence of food allergies (FA) in children increased rapidly at the turn of the century. The EuroPrevall study identified Germany as a country with very high prevalence of FA at that time. Using two large German birth cohorts, we provide an update of the status quo 10 years later.

Methods: KUNO Kids and UIm SPATZ Health studies are two ongoing prospective birth cohorts. Information on FA was obtained by questionnaires at birth and after 6,12 , and 24 months. Univariable and multivariable logistic regression analyses were performed to investigate risk factors during pregnancy, birth, and early childhood.

Results: In 1139 and 1006 children from KUNO Kids and SPATZ, the point prevalence of parent-reported FA symptoms at the ages of 1 and 2 years was $13.2 \%(95 \% \mathrm{Cl}$ : 11.2-15.2) and $13.9 \%$ (95\% Cl: $11.5-17.2)$ in KUNO Kids. Doctor's diagnosed FA at 1 and 2 years was $2.4 \%(95 \% \mathrm{Cl}: 1.6-3.4)$ and $2.7 \%(95 \% \mathrm{Cl}: 1.2-4.3)$ in KUNO Kids and $2.3 \%$ (95\% Cl: $1.3-3.6)$ and $3 \%(95 \% \mathrm{Cl}: 2.0-4.5)$ in SPATZ. Cow's milk and citrus fruits were most frequently suspected by parents to cause FA symptoms. Atopy in the child was associated with a higher frequency of FA at any time, whereas atopy in first-degree relatives was only associated with FA at year 1 . Smoke exposure during pregnancy was a risk for FA at age 2 .
\end{abstract}

Abbreviations: BMI, Body mass index; Cl, Confidence interval; DBPCFC, Double-blind placebo-controlled food challenge; FA, Food allergy; GDM, Maternal gestational diabetes; OR, Odds ratio; SD, Standard deviation.

Jon Genuneit and Michael Kabesch contributed equally.

This is an open access article under the terms of the Creative Commons Attribution-NonCommercial License, which permits use, distribution and reproduction in any medium, provided the original work is properly cited and is not used for commercial purposes.

(c) 2021 The Authors. Pediatric Allergy and Immunology published by European Academy of Allergy and Clinical Immunology and John Wiley \& Sons Ltd. 
German Federal Ministry for Education and Research (NAMIBIO: 01EA2108B).

Editor: Rachel Peters
Conclusion: The prevalence of food allergy seems to have plateaued in the last 10 years in Germany. FA is often suspected by parents but only rarely diagnosed by oral food challenge. Risk factor analysis may help to establish personalized health approaches.

\section{KEYWORDS}

birth cohort, epidemiology, food hypersensitivity, infant

\section{1 | INTRODUCTION}

Within the last five decades of the 20th century, the prevalence of atopic diseases like asthma, atopic dermatitis, and allergic rhinitis increased dramatically in children across the world ${ }^{1-3}$ and remained high. ${ }^{4,5}$ Food allergy (FA) seems to be the epidemic of the new century with rapidly increasing prevalence rates, ${ }^{3}$ becoming a major health burden in many countries, especially in children. ${ }^{6,7}$ The EuroPrevall birth cohort is the first and largest study to investigate FA in Europe. ${ }^{8}$ When recruitment was done in 2005, Germany was identified as one of the European countries with the highest prevalence of FA. ${ }^{9,10}$ However, it remains unclear whether FA further increased since then and whether risk factors for the disease changed over time.

Therefore, we investigated the prevalence of FA in infants and young children approximately 10 years after the EuroPrevall study in two large and currently ongoing German birth cohorts, the KUNO Kids Health study (KUNO Kids) ${ }^{11}$ and the UIm SPATZ Health Study (SPATZ). ${ }^{12,13}$ Furthermore, we explored the role of factors potentially associated with FA at ages 1 and 2 years, such as medical features of pregnancy and birth, nutrition of the child, and environmental exposures during pregnancy and infancy ${ }^{14-16}$ in KUNO Kids and sought for replication in SPATZ. Finally, we compared results to extractable EuroPrevall data.

\section{2 | METHODS}

\section{1 | Description of populations}

\section{\begin{tabular}{l|l} 
2.1.1 & The KUNO Kids Health study
\end{tabular}}

KUNO Kids is an ongoing prospective birth cohort started in 2015 which aims to explore the determinants of child health in a holistic approach. Recruitment is still ongoing. For the purpose of this study, we used data from the first and second phase of the study, which comprises 3100 newborns and their families recruited between June 2015 and March 2019. Data captured by follow-up questionnaires received by March 2020 were included in statistical analysis.

The precise methodology and design of the study have been described elsewhere. ${ }^{11}$ Briefly, written informed consent was obtained from all eligible mothers who gave birth at the children's and women's hospital St. Hedwig in Regensburg. Exclusion criteria were

\section{Key messages}

In two ongoing prospective birth cohorts in Germany, the prevalence of parent-reported FA in Germany was assessed and did not increase further in comparison with the EuroPrevall study 10 years ago. Parents suspect a multitude of different foods to cause FA symptoms, and oral food challenge is seldom used to confirm the diagnosis. Risk factors for FA include atopy and smoke exposure, seem to be age specific, and could provide targets for personalized medicine approach.

Based on data from two current birth cohorts, we suggest that FA prevalence reached a plateau in Germany when compared to EuroPrevall data from 10 years ago. Numerous non-core foods contribute to FA nowadays and risk factors for FA may contribute differently to FA occurring at various time points over the course of infancy.

unavailability for informed consent (i.e., insufficient German language skills) and mother's age below the legal age of 18 . The study design and procedures were approved by the ethics committee of the University of Regensburg (file number: 14-101-0347). An interview was conducted within days after birth, and follow-up questionnaires were sent to the families after 4 weeks, 6 months, and on every birthday of the child to collect additional information on the index child and the family. Socio-demographic information, medical history, feeding practices, lifestyle behaviors (nutrition, physical activity, smoking, alcohol consumption), and psychosocial constructs were collected from parents and siblings. Where available, validated questions and measurement instruments were used to allow for joint analyses of data with pre-existing studies, including SPATZ.

In KUNO Kids, parent-reported FA was defined in concordance with the EuroPrevall (using the same questionnaire) as a positive history of gastrointestinal, dermatological, or respiratory symptoms, which appeared in timed correlation to the food intake without other explanations for the symptoms. Gastrointestinal symptoms were nausea, vomiting, diarrhea, abdominal pain, constipation, and soft stools. Dermatological reactions comprised urticaria, angioedema, itching, and the appearance or worsening of eczema. Respiratory symptoms were defined as wheezing, dyspnea, coughing, stridor, and allergic rhinitis symptoms. Information on signs, symptoms, point of time, and clinical diagnostics of FA was 
collected using a validated questionnaire at 6 months and 1 and 2 years postpartum. Additional information on lifestyle-related factors like tobacco exposure during pregnancy, birth mode, infant feeding practices, environmental exposures during infancy, maternal and infant antibiotic intake as well as atopic diseases within the family was obtained from the KUNO Kids database. Parent-reported FA in KUNO Kids was defined as a parent report of FA-related symptoms in the past 12 months, while the doctor-reported FA was defined as a positive report of a doctor's diagnosis of FA at 1 and 2 years.

\subsection{2 | The Ulm SPATZ Health Study}

The UIm SPATZ Health Study is an ongoing birth cohort study. A total of 1006 newborns and their 970 mothers were enrolled into the study from the general population soon after delivery at the University Medical Centre Ulm, between April 2012 and May 2013. A detailed description of the study can be found elsewhere. ${ }^{12,13}$ Mothers were excluded from the study if (i) their German language skills were insufficient to understand the study procedure and consent forms, (ii) they had outpatient childbirth, (iii) they were $<18$ years of age, (iv) there was postpartum transfer of mother or child to an intensive care unit, or stillbirth. Participation in the study was entirely voluntary after informed consent was given. The study was approved by the Ethics board of UIm University (no. 311/11).

Demographic, lifestyle, and birth-related data including child gender, delivery mode, birthweight, maternal age, education, maternal body mass index (BMI), smoking status (within 1 year prior to delivery), maternal, paternal, and child history of allergic disease were collected using self-administered questionnaires, hospital electronic charts, and paperdocumented routine screening during pregnancy. Smoking status and alcohol consumption were assessed and defined as previously explained. ${ }^{12}$

A reported doctor's diagnosis of FA in the past 12 months was assessed at 1 year, 2 years, and at each further yearly follow-up by separate self-administered questionnaires from parents and the children's primary care pediatricians. In this case, the reports were distinguished between "parent-reported" FA and "pediatricianreported" FA; however, both were reports of a doctor's diagnosis. The parent-reported FA in SPATZ equals the doctor's diagnosed FA in the KUNO Kids study. FA symptoms were not assessed in SPATZ.

\section{2 | Statistical analysis}

The statistical analysis was conducted using Statistical Package for Social Sciences ${ }^{\circledR}$ (SPSS.24) and SAS 9.4 (The SAS ${ }^{\circledR}$ Institute). $p$-values $<.05$ were considered statistically significant. Descriptive statistics are displayed as median and interquartile range and mean and standard deviation for non-parametric and parametric data, respectively. First, univariable logistic regression analyses were used to explore the association of the different previously suggested risk factors with FA at age 1 and at age 2, in the KUNO Kids and in the UIm SPATZ cohort, respectively. Then, multivariable regression modeling was performed, for the respective FA outcome at age 1 and at age 2 in both cohorts. Risk factors were included in the multivariable models if they were associated with the outcome $(p<.2)$ at age 1 or at age 2 in the respective cohort. Odds ratios (OR) and 95\% confidence intervals $(\mathrm{Cl})$ are displayed.

\section{3 | RESULTS}

Of the 3100 newborns and their families enrolled in the KUNO Kids study until March 2020, 2679 were at least one year old when the dataset for this analysis was closed. FA data at one or more time points (6 months, 1 year, and 2 years) were available from 1139 infants. Compared with the total study population, cesarean section, having older siblings, exposure to household smoking, and a migration background were less frequent in children with completely available FA questionnaires until 1 year of age, while older mothers and a higher educational level were more frequent (Table 1 and Table S1).

Cumulatively, $23.9 \%$ of parents in the KUNO Kids study reported that their child had an adverse reaction to food in the first 2 years of life, not distinguishing between IgE- and non-IgE-mediated selfreported food allergy. The point prevalence was slightly higher at age 6 months and gastrointestinal and dermatological symptoms were most frequent (Figure 1). Cow's milk, citrus fruits, and wheat were most frequently described by parents as allegedly causative, while eggs were mentioned less frequently (Figure 2). In infants younger than 4 months, parents mentioned cow's milk most often to be responsible for the symptoms and fruits in 4 to 6 months old infants. (Figure 2). Some parents (143/1137) reported that a broad variety of fruits and vegetables not listed on the questionnaire (non-core foods) caused putative FA symptoms. For more than half $(59.7 \%$ ) of the children with reported FA at 6 months, no symptoms at the age of 1 year were reported. Of those with suspected FA at age 1 year, $58.1 \%$ did not report FA at age 2 years. Only $1.9 \%, 2.4 \%$, and $2.7 \%$ of parent-reported FA were diagnosed by a doctor at 6 months, age 1 , and age 2 years, respectively. Diagnostic confirmation by oral food challenge was only obtained in $30.8 \%$ of the 6 months cases, in $21.1 \%$ of the 1 -year cases and $10 \%$ of the 2 -year cases.

In a univariate analysis, we analyzed risk factors for FA suggested by previous studies for their association with FA at age 1 (Table S2A) and age 2 (Table S2B) in our dataset. The risk of parent-reported FA symptoms at 1 and 2 years of age was significantly higher if the child had other atopic diseases at the time. The presence of atopic diseases in first-degree relatives was related to an increased risk for food allergy at age 1 year, but not at age 2 years. Children with low birthweight had a decreased risk for FA at age 2 years while animal contact of the child or the mother during pregnancy (apart from farms) increased the risk for FA at this time point. Of note, passive smoke exposure of the mother was a strong predictor for FA at age 2 years. Only at age 1 year, a non-significant trend toward an increased risk to develop FA symptoms was observed in children of obese mothers compared with children of normal weight mothers 


\begin{tabular}{|c|c|c|c|c|}
\hline & \multicolumn{2}{|c|}{$\begin{array}{l}\text { Total study population, } \% \\
\text { or mean } \pm \text { SD }\end{array}$} & \multicolumn{2}{|c|}{$\begin{array}{l}\text { Study sample with data } \\
\text { on FA, } \% \text { or mean } \pm \text { SD }\end{array}$} \\
\hline & & $n$ & & $n$ \\
\hline \multicolumn{5}{|l|}{ Child } \\
\hline Male sex & 51.2 & 2653 & 49.9 & 1134 \\
\hline Duration of pregnancy (week) & $39.5 \pm 1.6$ & 2625 & $39.6 \pm 1.6^{*}$ & 1128 \\
\hline Cesarean section & 28.6 & 2679 & 27.8 & 1139 \\
\hline Birthweight (g) & $3359 \pm 509$ & 2638 & $3371 \pm 500$ & 1130 \\
\hline Antibiotic use & 2.7 & 2600 & 2.2 & 1113 \\
\hline Older siblings & 43.7 & 2636 & $38.5^{*}$ & 1130 \\
\hline Migration background ${ }^{a}$ & 28.0 & 1824 & $14.2^{*}$ & 1065 \\
\hline \multicolumn{5}{|l|}{ Mother } \\
\hline Age (years) & $32.7 \pm 4.5$ & 2651 & $33.1 \pm 4.1^{*}$ & 1132 \\
\hline Pre-pregnancy BMI $\left(\mathrm{kg} / \mathrm{m}^{2}\right)$ & $23.8 \pm 5.0$ & 2614 & $23.7 \pm 4.7$ & 1121 \\
\hline Regular animal contact ${ }^{\mathrm{b}}$ & 54.9 & 2601 & 53.8 & 1116 \\
\hline $\begin{array}{l}\text { Higher educational level } \\
\qquad(>10 \text { years })\end{array}$ & 56.5 & 2597 & $62.8^{*}$ & 1116 \\
\hline Smokers in the household & 22.4 & 2601 & $17.0^{*}$ & 1114 \\
\hline
\end{tabular}

TABLE 1 Characteristics of the study population (KUNO Kids study)

Note: ${ }^{*}$ Comparison with total study population yielded statistically significant difference $(p<.001)$.

Abbreviations: BMI, body mass index; FA, food allergy; SD, standard deviation.

${ }^{a}$ Mother or father with non-German nationality or born not in Germany.

${ }^{\mathrm{b}}$ Regular contact (more than once per month) to any pet during pregnancy.

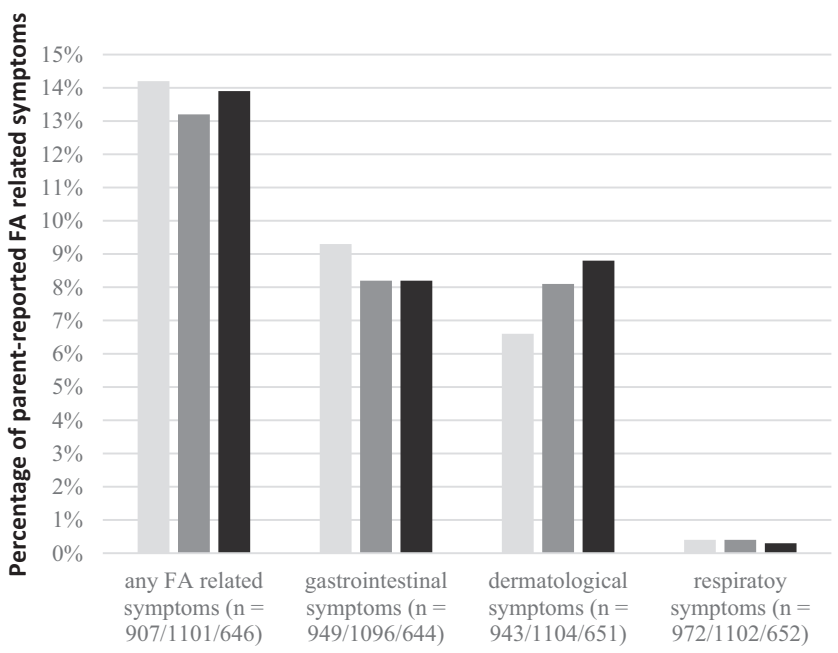

FA related symptoms at age:

- 6 months $\quad 12$ months $\quad 24$ months

FIGURE 1 Prevalence and characteristics of parent-reported food allergy related symptoms over the first 2 years in the KUNO Kids study

(measured before pregnancy). Of the 1006 infants enrolled into the SPATZ study, $4.1 \%$ of the infants had at least one food allergy reported either by the caring pediatrician or by the parent (parental report of the doctor's diagnosis) within the first year of life. Common risk factors for FA were also investigated in the SPATZ study at age 1 and 2 years, whenever available (Table S3A and B). Again, the presence of other allergies in the child was statistically significantly associated with FA (years 1 and 2) and atopic diseases in first-degree relatives were a significant predictor for FA at age 1 year.

Next, we performed multiple regression modeling for the respective FA outcomes at age 1 and at age 2 in both cohorts based on risk factors which $p<.2$ in the univariate analyses. For KUNO Kids, atopic disease in the child was strongly associated with FA at age 1 and 2 years while a positive family history was associated only with FA at age 1 year (Table 2A and B). Gestational diabetes in the mother and exposure to traffic showed suggestive associations $(p<.1)$ at age 1 and 2 years, respectively. Multivariate regression in the SPATZ study confirmed the association between another atopic disease in the child and FA at both ages (Table $3 \mathrm{~A}$ and B). Furthermore, associations between male gender for FA at age 1 and exposure to cigarette smoking with FA at age 1 (suggestive) and age 2 (significant) were identified.

Finally, we compared the data from the KUNO Kids and SPATZ studies with results from EuroPrevall acquired approximately 10 years earlier. (Table 4). On average, mothers in the KUNO Kids study were approximately one to two years older when compared to mothers from the EuroPrevall and SPATZ studies. The frequency of cesarean section birth was lower in the KUNO Kids and SPATZ studies, while antibiotic use during delivery was considerably higher in KUNO Kids compared with EuroPrevall and SPATZ. Also, the prevalence of maternal smoking and passive smoking varied significantly between studies. Data on overall parent-reported and doctor-diagnosed FA frequencies in EuroPrevall were extracted from the manuscript by Schoemaker et al. ${ }^{17}$ Frequencies were in a similar range in the two recent birth cohort studies KUNO Kids and 
FIGURE 2 First occurrence of parent-reported adverse reactions to food allergens in the first 2 years of life $(n=189)$ and proportion of core foods causing the adverse reactions reported for specific months in the KUNO Kids study
35

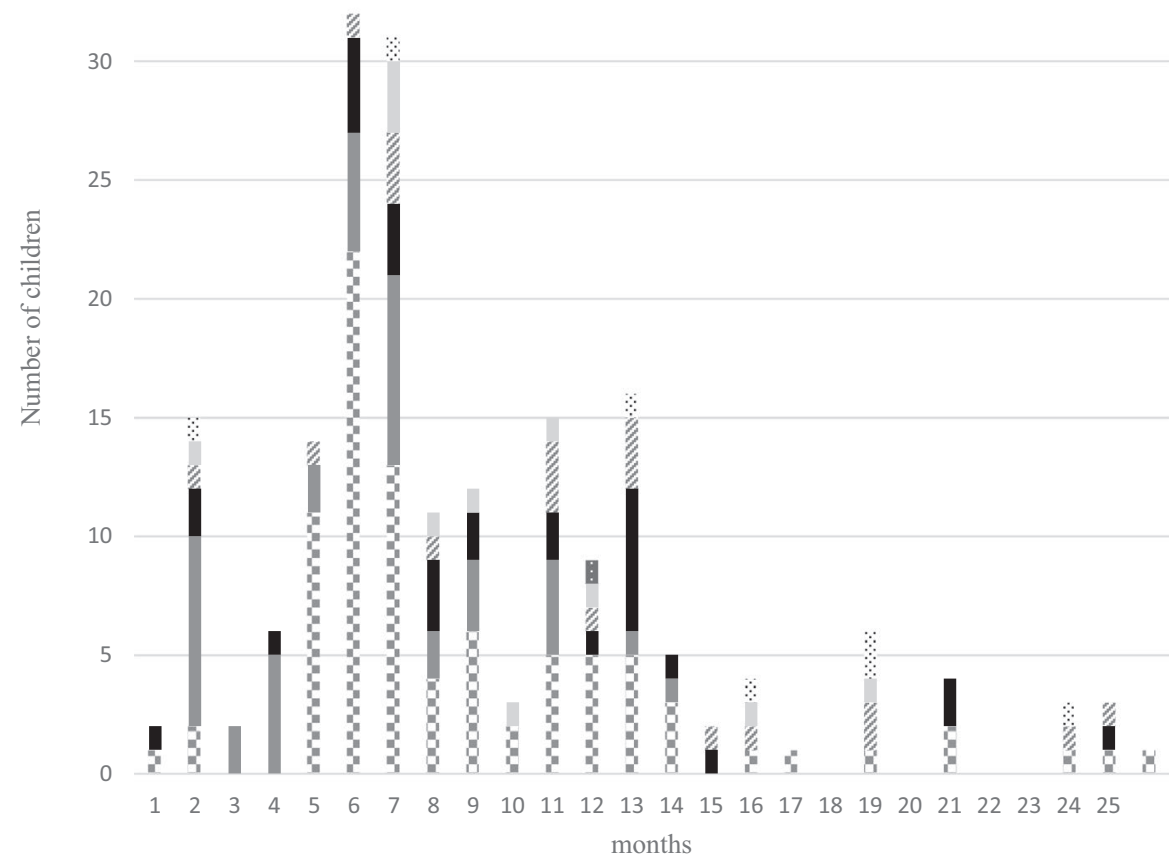

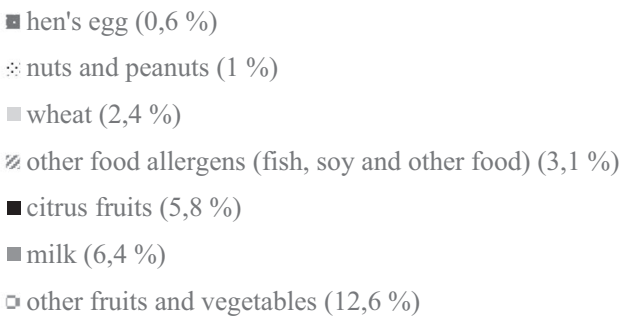

SPATZ as well as the reported German and overall EuroPrevall data for parent-reported FA symptoms and doctor's diagnosis of FA at age 2 years.

\section{4 | DISCUSSION}

Data from KUNO Kids and SPATZ show that the prevalence of parent-reported FA and doctor's diagnosis of FA in infants at age 1 and 2 years did not differ substantially from results obtained by the EuroPrevall study 10 years ago. Non-core foods seem to play an important role in FA now. When classic risk factors for FA were investigated in the current studies, family history for atopy was associated with FA at age 1 but less so at age 2, while maternal passive smoke exposure was a risk factor for FA at age 2. Our study demonstrated that FA seems to be a highly dynamic entity with various changes in point prevalence, risk factors, and predictors over the first 2 years of life.

Some differences between study populations were observed, but most of the factors that have been associated with an increased risk of FA in previous studies were more pronounced in KUNO Kids and SPATZ. Due to the population structure of KUNO Kids with older, highly educated mothers, an over-reporting of food allergy symptoms may be expected, and consequently, FA would be overestimated. However, compared with data available in EuroPrevall at age 2 years for Germany and Europe, no clear difference was observed. Even though methodology differed, and the two study regions investigated here are Regensburg (KUNO Kids) and UIm (SPATZ) and not Berlin (EuroPrevall), results seem comparable enough to assess trends over time. For the prevalence of doctor's diagnosis of food allergy, reports at age 1 and 2 years are very similar for KUNO Kids and SPATZ (Table 3). For EuroPrevall, prevalence data for Europe and Germany were taken from the publication of Schoemaker. ${ }^{17}$ Since the focus of that study is on cow's milk allergy, the calculated rate of doctor's diagnosed FA may be underestimated for EuroPrevall Germany. Taking all these factors into account, there is no indication of a significant rise in the prevalence of FA in Germany over the last 10 years. This is of interest, because at the turn of the century a worldwide trend toward an increasing FA prevalence was suggested $^{6}$ when hospital admissions for food allergy quintupled ${ }^{3}$ and the number of children with FA increased by twelve times between 1995 and $2006 .^{2}$ Especially in children below 2 years, an increase in food-induced anaphylaxis was reported. ${ }^{7}$ Our data now suggest that the prevalence of food allergies reached a plateau in Germany, similar to what has been observed for many other atopic diseases, such as asthma, years earlier. ${ }^{18-20}$ 
TABLE 2 Multivariable logistic regression analysis in KUNO Kids of potential risk factors: Outcome parent-reported symptoms of FA

\begin{tabular}{|c|c|c|}
\hline & Odds ratio $(95 \% \mathrm{Cl})$ & $\begin{array}{l}p \\
\text { Value }\end{array}$ \\
\hline \multicolumn{3}{|l|}{ A) At age 1} \\
\hline \multicolumn{3}{|l|}{ Child } \\
\hline Cesarean section & $0.828(0.562-1.219)$ & .339 \\
\hline Birthweight $<2800 \mathrm{~g}$ & $0.817(0.466-1.432)$ & .480 \\
\hline $\begin{array}{l}\text { Duration of breastfeeding } \\
<6 \text { month }\end{array}$ & $0.920(0.640-1.322)$ & .653 \\
\hline Daily animal contact ${ }^{\mathrm{a}}$ & $1.091(0.725-1.642)$ & .676 \\
\hline $\begin{array}{l}\text { High exposure to traffic, } \\
\text { exhaust gases }\end{array}$ & $1.038(0.739-1.459)$ & .828 \\
\hline Other atopic diseases ${ }^{b}$ & $3.681(2.504-5.412)$ & $<.001$ \\
\hline \multicolumn{3}{|l|}{ Mother } \\
\hline \multicolumn{3}{|l|}{ Pre-pregnancy BMI } \\
\hline Underweight & $1.894(0.637-5.633)$ & .251 \\
\hline Normal weight & reference & \\
\hline Overweight & $1.041(0.692-1.571)$ & .841 \\
\hline Obese & $1.377(0.822-2.305)$ & .224 \\
\hline Gestational diabetes & $1.503(0.960-2.354)$ & .075 \\
\hline $\begin{array}{l}\text { Passive exposure to } \\
\text { cigarette smoking }\end{array}$ & $1.049(0.689-1.598)$ & .823 \\
\hline Regular animal contact ${ }^{c}$ & $0.969(0.641-1.466)$ & .883 \\
\hline \multicolumn{3}{|l|}{ School education } \\
\hline Low (no degree) & $1.463(0.721-2.969)$ & .292 \\
\hline Intermediate ( $<10$ years) & reference & \\
\hline High (>10 years) & $1.120(0.755-1.662)$ & .572 \\
\hline $\begin{array}{l}\text { Atopic disease of first- } \\
\text { degree relatives }\end{array}$ & $1.531(1.042-2.250)$ & .030 \\
\hline \multicolumn{3}{|l|}{ B) At age 2} \\
\hline \multicolumn{3}{|l|}{ Child } \\
\hline Cesarean section & $0.875(0.482-1.587)$ & .659 \\
\hline Birthweight $<2800 \mathrm{~g}$ & $0.425(0.141-1.277)$ & .127 \\
\hline $\begin{array}{l}\text { Duration of breastfeeding } \\
\quad<6 \text { month }\end{array}$ & $0.832(0.478-1.450)$ & .517 \\
\hline Daily animal contacts ${ }^{a}$ & $1.685(0.894-3.177)$ & .107 \\
\hline $\begin{array}{l}\text { High exposure to traffic, } \\
\text { exhaust gases }\end{array}$ & $1.608(0.953-2.712)$ & .075 \\
\hline Other atopic diseases ${ }^{\mathrm{b}}$ & $2.705(1.472-4.972)$ & .001 \\
\hline \multicolumn{3}{|l|}{ Mother } \\
\hline \multicolumn{3}{|l|}{ Pre-pregnancy BMI } \\
\hline Underweight & $-/-($ no cases with FA) & $-1-$ \\
\hline Normal weight & reference & \\
\hline Overweight & $1.305(0.707-2.409)$ & .395 \\
\hline Obese & $1.107(0.506-2.420)$ & .799 \\
\hline Gestational diabetes & $0.569(0.240-1.347)$ & .199 \\
\hline $\begin{array}{l}\text { Passive exposure to } \\
\text { cigarette smoking }\end{array}$ & $1.569(0.878-2.804)$ & .128 \\
\hline Regular animal contact ${ }^{c}$ & $1.318(0.697-2.492)$ & .395 \\
\hline
\end{tabular}

TABLE 2 (Continued)

\begin{tabular}{|c|c|c|}
\hline & Odds ratio $(95 \% \mathrm{Cl})$ & $\begin{array}{l}p \\
\text { Value }\end{array}$ \\
\hline \multicolumn{3}{|l|}{ School education } \\
\hline Low (no degree) & $1.999(0.722-5.535)$ & .182 \\
\hline Intermediate ( $<10$ years) & reference & \\
\hline High (>10 years) & $1.081(0.576-2.028)$ & .808 \\
\hline $\begin{array}{c}\text { Atopic disease of first- } \\
\text { degree relatives }{ }^{d}\end{array}$ & $1.470(0.809-2.669)$ & .206 \\
\hline
\end{tabular}

Note: Risk factors were included if they were associated with the 1-year or the 2-year outcome $(p<.2)$ in univariable analysis; (A) $N=909$; $R^{2}: .103$; (B) $N=509 ; R^{2}: .123$.

Abbreviations: $95 \% \mathrm{Cl}, 95 \%$ confidence interval; BMI, body mass index; FA, food allergy.

${ }^{a}$ Daily contact to dog, cat, hamster, guinea pig, mouse, rat, or rabbit.

${ }^{b}$ Atopic eczema, allergic rhinitis, allergic conjunctivitis, or asthma.

${ }^{\mathrm{c}}$ Regular contact (more than once per month) to any pet during pregnancy.

${ }^{\mathrm{d}}$ Atopic eczema, allergic rhinitis, allergic conjunctivitis, asthma, or food allergy in parents or blood-related siblings.

TAB LE 3 Multivariable logistic regression analysis in Ulm SPATZ Health Study of potential risk factors: Outcome parent-reported FA

Odds ratio $(95 \% \mathrm{Cl}) \quad p$ Value

\section{A) At age 1 \\ Child}

\begin{tabular}{|c|c|c|}
\hline Male sex & $1.909(1.003-13.223)$ & .049 \\
\hline Other atopic diseases ${ }^{a}$ & $2.99(3.037-26.321)$ & $<.001$ \\
\hline \multicolumn{3}{|l|}{ Mother } \\
\hline Gestational diabetes & $1.677(0.699-11.303)$ & .145 \\
\hline $\begin{array}{l}\text { Cigarette smoking during } \\
\text { pregnancy }\end{array}$ & $1.572(0.852-7.174)$ & .096 \\
\hline $\begin{array}{l}\text { Antibiotic use during } \\
\text { pregnancy }\end{array}$ & $1.513(0.673-7.777)$ & .185 \\
\hline $\begin{array}{l}\text { Atopic disease of first- } \\
\text { degree relatives }^{\mathrm{b}}\end{array}$ & $1.844(0.932-12.413)$ & .064 \\
\hline
\end{tabular}

B) At age 2

Child

$\begin{array}{lll}\text { Male sex } & 1.443(0.841-5.16) & .113 \\ \text { Other atopic diseases }^{\mathrm{a}} & 2.012(1.639-10.01) & .002\end{array}$

Mother

Gestational diabetes $\quad 1.279(0.456-5.876) \quad .450$

Cigarette smoking during $\quad 1.627(1.099-6.371) \quad .030$ pregnancy

Antibiotic use during $\quad 1.235(0.493-4.716) \quad .464$ pregnancy

Atopic disease of first- $\quad 1.417(0.807-4.998) \quad .134$ degree relatives ${ }^{b}$

Note: Risk factors were included if they were associated with the 1-year or the 2-year outcome $(p<.2)$ in univariable analysis; (A) $N=729$; $R^{2}:$.044; (B) $N=788 ; R^{2}: .026$.

Abbreviations: 95\% $\mathrm{Cl}-95 \%$ confidence interval; FA-food allergy.

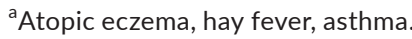

${ }^{\mathrm{b}}$ Atopic eczema, hay fever, asthma in parents and blood-related siblings. 
TAB LE 4 Comparison of study results from KUNO Kids (recruitment 2015-2019), SPATZ (2012-2013) and EuroPrevall (2005-2010)

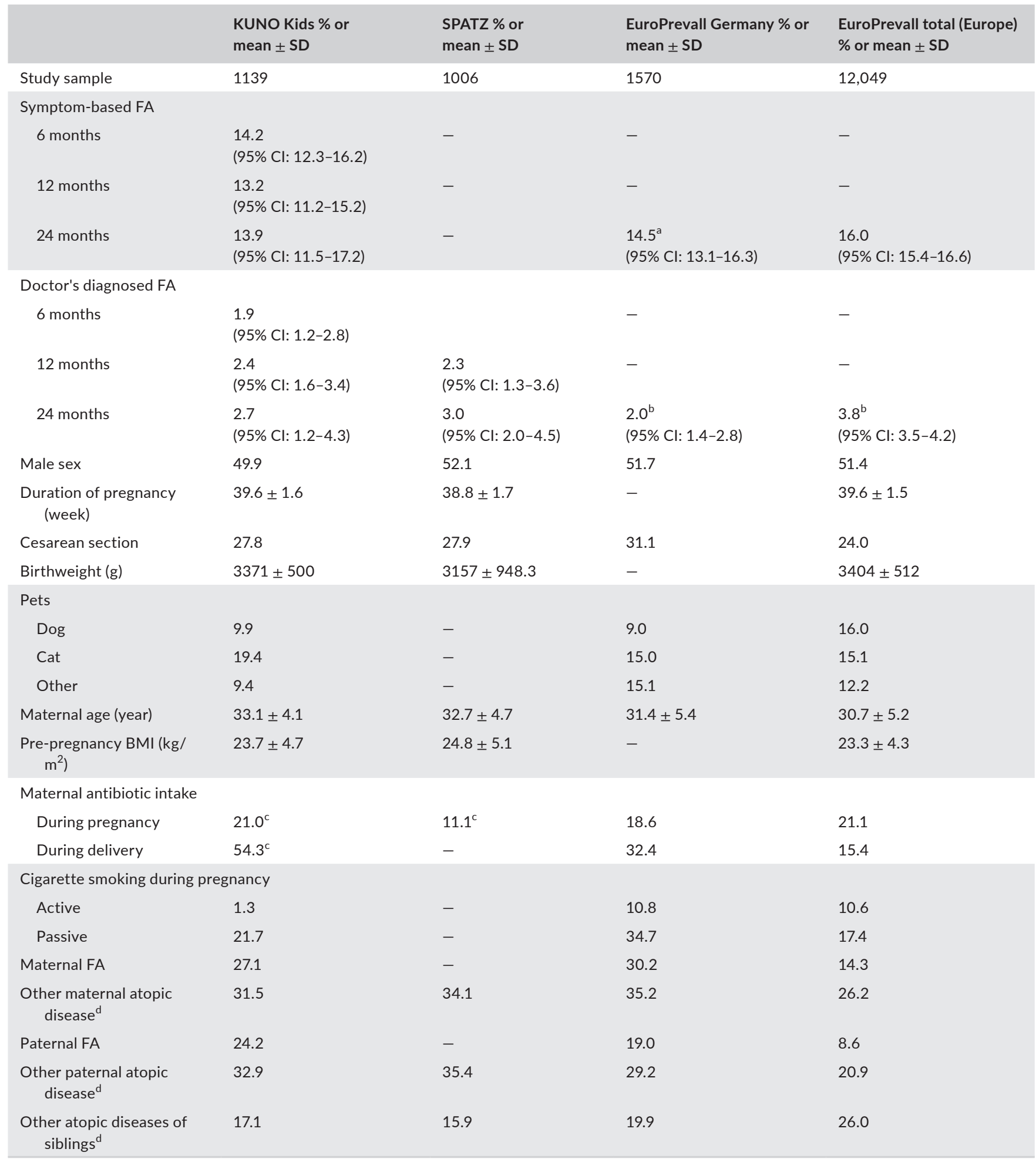

Abbreviations: $95 \% \mathrm{Cl}$, 95\% confidence interval; $\mathrm{BMI}$, body mass index; FA, food allergy; SD, standard deviation.

${ }^{a}$ EuroPrevall documented this frequency for self-reported FA in siblings of their study children (Schoemaker et al., 2015, Allergy). ${ }^{17}$

${ }^{b}$ Calculated from data from Schoemaker et al., 2015, Allergy, ${ }^{17}$ as percentage of children eligible for DBPCFC.

'Different methods: KUNO kids used a specific question ("Did you take any antibiotics during pregnancy/delivery?"), SPATZ used an open question after delivery about any medication intake during pregnancy.

${ }^{\mathrm{d}}$ Atopic eczema, allergic rhinitis, allergic conjunctivitis, or asthma. 
In KUNO Kids and SPATZ, assessments of FA were based on questionnaires only, while in EuroPrevall, questionnaires and a double-blind placebo-controlled food challenge (DBPCFC) were applied. Several studies suggest that the prevalence of parentreported FA overestimates the actual FA prevalence. ${ }^{10,21,22}$ In our study, parents suspected a multitude of different foods, especially fruit and vegetables, to be responsible for adverse reactions. While known allergens like cow's milk, wheat, hen's egg, soy, and peanut are responsible for IgE-mediated allergy, these putative causative agents seem to represent a fear of parents rather than actual food allergy. Citrus fruit and histamine-rich foods can cause perioral itching and redness (especially in children with eczema) which is not due to IgE-mediated allergy. Further investigations into that observation are needed.

The results of KUNO Kids showed that FA in infants is often not diagnosed with DBPCFC: two-thirds at the age of 6 months, $79 \%$ at the age of 1 year, and $90 \%$ at the age of 2 years were diagnosed with other methods than DBPCFC which suggests that better education of doctors and parents along with increasing resources for DBPCFC may be necessary and needed to avoid unnecessary and even harmful dietary restrictions in children based on FA assumptions. However, results can be comparable between studies for questionnaire-derived data, especially as KUNO Kids used the same questions as EuroPrevall (for symptoms of FA) and SPATZ (for doctor-diagnosed FA). Simple, questionnaire-based assessment tools are key for replication and comparisons between populations as previously demonstrated by the worldwide ISAAC project. $^{23}$

Some previously suggested risk factors ${ }^{24-26}$ were assessed here by univariate analysis in KUNO Kids and SPATZ and followed up in a multivariate analysis. Interestingly, a family history of atopic diseases in first-degree relatives was associated with an increased risk for FA at age 1 but not age 2 in both studies. This may either indicate that the genetic impact on FA decreases with age or that pathogenesis of food allergy at age 2 years differs from FA at age 1 year. Accordingly, we demonstrated an extensive turnaround in presence and absence of FA symptoms over that time span. A (changeable) risk factor for FA at age 2, but less so at age 1 , is passive smoke exposure either through maternal smoking during pregnancy or via postnatal environmental exposure in both birth cohorts. This may present a valuable opportunity for future efforts of intervention and prevention.

In conclusion, our study suggests that the prevalence of FA in Germany has reached a plateau in infants and young children. Numerous allergens other than core foods are associated with FA in infants now. While better diagnosis of FA with DBPCFC in clinical practice is needed, questionnaire data can provide valuable comparisons between different populations and inform about time trend and regional differences. Risk factors for the development of FA seem to be age specific and that needs to be taken into account more carefully for further FA prediction analysis, when personalized medicine may be applied to FA.

\section{ACKNOWLEDGMENTS}

We are grateful to all participants in the KUNO Kids and the UIm SPATZ Health Studies and to their parents for filling out questionnaires over years. We thank all the students who contributed time and effort in the collection of data and all the partners in the study for their input and support.

\section{CONFLICT OF INTEREST}

D. Rothenbacher received research grants from Danone Nutricia Research, and J. Genuneit is the project manager of research grants from Danone to both Ulm University and Leipzig University in relation to studies of the composition of breastmilk including data of the UIm SPATZ Health Study. All authors declare that they have no competing financial or personal interests.

\section{AUTHOR CONTRIBUTIONS}

Daniela Verena Roehrl: Investigation (equal); Writing-original draft (lead). Susanne Brandstetter: Data curation (equal); Formal analysis (lead); Validation (equal); Writing-original draft (supporting). Linda P. Siziba: Data curation (equal); Formal analysis (equal); Investigation (supporting); Writing-original draft (supporting). Dietrich Rothenbacher: Data curation (supporting); Funding acquisition (supporting); Methodology (supporting); Writing-review \& editing (supporting). Vincent David Gaertner: Data curation (supporting); Formal analysis (supporting); Investigation (supporting); Validation (supporting); Writing-review \& editing (supporting). Susanne Harner: Data curation (supporting); Methodology (supporting); Validation (supporting); Writing-review \& editing (supporting). Angela Köninger: Data curation (supporting); Funding acquisition (supporting); Writing-review \& editing (supporting). Christian Apfelbacher: Data curation (supporting); Formal analysis (supporting); Methodology (equal); Validation (lead); Writingreview \& editing (equal). Michael Melter: Data curation (supporting); Funding acquisition (supporting). Jon Genuneit: Conceptualization (supporting); Data curation (equal); Formal analysis (equal); Funding acquisition (equal); Investigation (equal); Project administration (supporting); Resources (equal); Supervision (equal); Writing-review \& editing (equal). Michael Kabesch: Conceptualization (lead); Data curation (equal); Formal analysis (equal); Funding acquisition (lead); Investigation (lead); Methodology (lead); Project administration (lead); Supervision (lead); Writing-review \& editing (lead).

\section{PEER REVIEW}

The peer review history for this article is available at https://publo ns.com/publon/10.1111/pai.13689.

\section{ORCID}

Linda P. Siziba (ID) https://orcid.org/0000-0002-4773-252X

Susanne Harner (D) https://orcid.org/0000-0001-6733-5823

Christian Apfelbacher (D) https://orcid.org/0000-0003-3805-8219

Jon Genuneit (D) https://orcid.org/0000-0001-5764-1528

Michael Kabesch (D) https://orcid.org/0000-0003-0697-1871 


\section{REFERENCES}

1. Pearce N, Aït-Khaled N, Beasley R, et al. Worldwide trends in the prevalence of asthma symptoms: phase III of the International Study of Asthma and Allergies in Childhood (ISAAC). Thorax. 2007;62(9):758-766. doi:10.1136/thx.2006.070169

2. Mullins RJ. Paediatric food allergy trends in a communitybased specialist allergy practice, 1995-2006. Med J Aust. 2007;186(12):618-621.

3. Gupta R, Sheikh A, Strachan DP, Anderson HR. Time trends in allergic disorders in the UK. Thorax. 2007;62(1):91-96. doi:10.1136/ thx.2004.038844

4. Schuh C, Fritscher LG, Chapman KR, Fritscher CC. The prevalence of asthma and atopy in schoolchildren from Porto Alegre, Brazil, has plateaued. Respir Med. 2015;109(3):308-311. doi:10.1016/j. rmed.2015.01.014

5. Braun-Fahrländer C, Gassner M, Grize L, et al. No further increase in asthma, hay fever and atopic sensitisation in adolescents living in Switzerland. Eur Respir J. 2004;23(3):407-413. doi:10.1183/09031 936.04.00074004

6. Prescott SL, Pawankar R, Allen KJ, et al. A global survey of changing patterns of food allergy burden in children. World Allergy Organ J. 2013;6(1):21. doi:10.1186/1939-4551-6-21

7. Motosue MS, Bellolio MF, van Houten HK, Shah ND, Campbell RL. National trends in emergency department visits and hospitalizations for food-induced anaphylaxis in US children. Pediatr Allergy Immunol. 2018;29(5):538-544. doi:10.1111/pai.12908

8. McBride D, Keil T, Grabenhenrich L, et al. The EuroPrevall birth cohort study on food allergy: baseline characteristics of 12,000 newborns and their families from nine European countries. Pediatr Allergy Immunol. 2012;23(3):230-239. doi:10.1111/j.1399-3038.2011.01254.x

9. Burney P, Summers C, Chinn S, Hooper R, van Ree R, Lidholm J. Prevalence and distribution of sensitization to foods in the European Community Respiratory Health Survey: a EuroPrevall analysis. Allergy. 2010;65(9):1182-1188. doi:10.1111/j.1398-9995.2010.02346.x

10. Xepapadaki P, Fiocchi A, Grabenhenrich L, et al. Incidence and natural history of hen's egg allergy in the first 2 years of lifethe EuroPrevall birth cohort study. Allergy. 2016;71(3):350-357. doi:10.1111/all.12801

11. Brandstetter S, Toncheva AA, Niggel J, et al. KUNO-Kids birth cohort study: rationale, design, and cohort description. Mol Cell Pediatr. 2019;6(1):1. doi:10.1186/s40348-018-0088-z

12. Logan C, Zittel T, Striebel S, et al. Changing societal and lifestyle factors and breastfeeding patterns over time. Pediatrics. 2016;137(5):e20154473. doi:10.1542/peds.2015-4473

13. Braig S, Weiss JM, Stalder T, Kirschbaum C, Rothenbacher D, Genuneit J. Maternal prenatal stress and child atopic dermatitis up to age 2 years: the UIm SPATZ health study. Pediatr Allergy Immunol. 2017;28(2):144-151. doi:10.1111/pai.12680

14. Gupta M, Sicherer SH. Timing of food introduction and atopy prevention. Clin Dermatol. 2017;35(4):398-405. doi:10.1016/j.clind ermatol.2017.03.013

15. van Ginkel CD, van der Meulen GN, Bak E, et al. Retrospective observational cohort study regarding the effect of breastfeeding on challenge-proven food allergy. Eur J Clin Nutr. 2018;72(4):557-563. doi:10.1038/s41430-018-0117-y

16. Ben-Shoshan M, Soller L, Harrington DW, et al. Eczema in early childhood, sociodemographic factors and lifestyle habits are associated with food allergy: a nested case-control study. Int Arch Allergy Immunol. 2015;166(3):199-207. doi:10.1159/000381829

17. Schoemaker AA, Sprikkelman AB, Grimshaw KE, et al. Incidence and natural history of challenge-proven cow's milk allergy in European children-EuroPrevall birth cohort. Allergy. 2015;70(8):963-972. doi:10.1111/all.12630

18. Prescott S, Allen KJ. Food allergy: riding the second wave of the allergy epidemic. Pediatr Allergy Immunol. 2011;22(2):155-160. doi:10.1111/j.1399-3038.2011.01145.x

19. Asher MI, Montefort S, Björkstén B, et al. Worldwide time trends in the prevalence of symptoms of asthma, allergic rhinoconjunctivitis, and eczema in childhood: ISAAC Phases One and Three repeat multicountry cross-sectional surveys. Lancet. 2006;368(9537):733743. doi:10.1016/S0140-6736(06)69283-0

20. Björkstén B, Clayton T, Ellwood P, Stewart A, Strachan D. Worldwide time trends for symptoms of rhinitis and conjunctivitis: Phase III of the International Study of Asthma and Allergies in Childhood. Pediatr Allergy Immunol. 2008;19(2):110-124. doi:10.1111/j.1399-3038.2007.00601.x

21. Venter C, Pereira B, Grundy J, et al. Incidence of parentally reported and clinically diagnosed food hypersensitivity in the first year of life. J Allergy Clin Immunol. 2006;117(5):1118-1124. doi:10.1016/j. jaci.2005.12.1352

22. Nwaru BI, Hickstein L, Panesar SS, Roberts G, Muraro A, Sheikh A. Prevalence of common food allergies in Europe: a systematic review and meta-analysis. Allergy. 2014;69(8):992-1007. doi:10.1111/ all.12423

23. Flohr C, Weinmayr G, Weiland SK, et al. How well do questionnaires perform compared with physical examination in detecting flexural eczema? Findings from the International Study of Asthma and Allergies in Childhood (ISAAC) Phase Two. Br J Dermatol. 2009;161(4):846-853. doi:10.1111/j.1365-2133.2009.09261.x

24. Koplin JJ, Dharmage SC, Ponsonby A-L, et al. Environmental and demographic risk factors for egg allergy in a population-based study of infants. Allergy. 2012;67(11):1415-1422. doi:10.1111/ all.12015

25. Koplin JJ, Peters RL, Ponsonby A-L, et al. Increased risk of peanut allergy in infants of Asian-born parents compared to those of Australian-born parents. Allergy. 2014;69(12):1639-1647. doi:10.1111/all.12487

26. Koplin JJ, Allen KJ, Tang MLK. Important risk factors for the development of food allergy and potential options for prevention. Expert Rev Clin Immunol. 2019;15(2):147-152. doi:10.1080/17446 66X.2019.1546577

\section{SUPPORTING INFORMATION}

Additional supporting information may be found in the online version of the article at the publisher's website.

How to cite this article: Röhrl DV, Brandstetter S, Siziba LP, et al; the KUNO Kids Study Group. Food allergy in infants assessed in two German birth cohorts 10 years after the EuroPrevall Study. Pediatr Allergy Immunol. 2021;00:1-9. https://doi.org/10.1111/pai.13689 